\title{
RETRACTED ARTICLE: A single chain (scFv425):sTRAIL fusion protein with specificity for the EGF receptor is effective in vitro but not in an in vivo brain tumor animal model
}

\author{
Jos M. A. Kuijlen • Jan Jakob A. Mooij • \\ Wijnand Helfrich • Wilfred F. A. den Dunnen
}

Received: 31 October 2006/ Accepted: 15 December 2006

(C) Springer Science+Business Media B.V. 2007

\section{Retraction statement}

The article has been retracted by agreement between the authors and the editors. The reasons for retraction are twofold:

1 First author Mr Kuijlen failed to inform Dr. Helfrich on the contents of the manuscript, on the fact it was submitted for publication, and that Dr. Helfrich was listed as an author.

2 Only after online publication Dr. Helfrich became aware of this work in which he immediately detected mistakes in Mr Kuijlen's assumptions and calculations.
All listed authors agree on the fact that the conclusion (also given in the title) about inactivity of the scFv425:sTRAIL fusion protein in vivo is unjustified. Therefore this publication should be ignored. Mr Kuijlen apologizes to all authors and to the editors and readership of the Journal of Neurooncology.

The article has been retracted by agreement between the authors and the editors. The reasons for retraction are specified in the retraction statement that replaces the original publication.

J. M. A. Kuijlen $(\bowtie) \cdot$ J. J. A. Mooij

Department of Neurosurgery, University Medical Center

Groningen, University Hospital Groningen, Hanzeplein 1, P.O. Box 30.001, 9700 RB Groningen, The Netherlands

e-mail: j.m.a.kuijlen@nchir.umcg.nl

W. Helfrich

Department of Pathology and Laboratory Medicine,

Section Medical Biology, Laboratory for Tumor Immunology,

University Medical Center Groningen, University Hospital

Groningen, Hanzeplein 1, P.O. Box 30.001, 9700 RB Groningen,

The Netherlands

W. F. A. den Dunnen

Department of Pathology and Laboratory Medicine,

University Medical Center Groningen, University Hospital

Groningen, Hanzeplein 1, P.O. Box 30.001,

9700 RB Groningen, The Netherlands 